\section{Statuten der Schweizerischen Gesellschaft für Dermatologie und Venerologie}

\section{Name, Sitz und Zweck}

\section{Art. 1 Name und Sitz}

${ }^{1}$ Unter dem Namen «Schweizerische Gesellschaft für Dermatologie und Venerologie», «Société suisse de dermatologie et vénéréologie», «Società svizzera di dermatologia e venereologia», «Societad svizzra da dermatologia e venerologia», «Swiss Society of Dermatology and Venereology» (SGDV) besteht ein Verein im Sinne von Art. 60ff. des Schweizerischen Zivilgesetzbuches. Die «Schweizerische Gesellschaft für Dermatologie und Syphiligraphie (Venerologie seit 1917)» wurde am 24. April 1913 in Genf gegründet.

${ }^{2}$ Der Verein hat seinen Sitz am Wohnort des Präsidenten.

${ }^{3}$ Sofern in diesen Statuten die männliche Form oder Bezeichnung verwendet wird, gilt die weibliche als eingeschlossen.

\section{Art. 2 Zweck}

${ }^{1}$ Als gesamtschweizerische Organisation und als Zweckvereinigung vertritt die SGDV die Dermatologen und die akademische Dermatologie in der Schweiz.

2 Die Vereinigung bezweckt die Interessenvertretung und Interessenwahrung der SGDV und der Mitglieder. Sie vertritt die Mitglieder gegenüber der Bevölkerung, den Behörden, den Krankenversicherern und weiteren Institutionen.

${ }^{3}$ Die SGDV bezweckt insbesondere:

a) Die Förderung des Spezialfaches Dermatologie und Venerologie, sowie ihrer Subspezialitäten, das als wesentliche Bereiche die Physiologie und Pathologie der Haut und ihrer Anhangsorgane, die sexuell übertragbaren Krankheiten sowie alle zur Behandlung von diesbezüglichen Affektionen vorliegenden wissenschaftlich geprüften diagnostischen und therapeutischen Verfahren umfasst.

b) Die Förderung und den Austausch wissenschaftlicher und praktischer Erfahrungen im Bereich der Dermatologie und Venerologie.

c) Die Weiter- und Fortbildung im Bereich der Dermatologie und Venerologie.

d) Die Wahrung der Standesinteressen.

e) Die Zusammenarbeit mit der Verbindung der Schweizer Ärzte (FMH), insbesondere im Rahmen der Weiter- und Fortbildungsordnung der FMH. dern.

f) Die Pflege freundschaftlicher Beziehungen unter den Mitglie-

${ }^{4}$ Die Gesellschaft verpflichtet sich und ihre Mitglieder zur Einhaltung der Statuten der Verbindung der Schweizer Ärzte und der verbindlichen Beschlüsse der Schweizerischen Ärztekammer.

\section{Mitgliedschaft}

Art. 3 Mitgliederkategorien

Es bestehen folgende Mitgliederkategorien:

- Ehrenmitglieder

- ordentliche Mitglieder

- ausserordentliche Mitglieder

- korrespondierende Mitglieder

- Passivmitglieder

\section{Art. 4 Ehrenmitglieder}

${ }^{1}$ Persönlichkeiten, die sich um die SGDV, die Medizin oder das Fach besonders verdient gemacht haben, können zum Ehrenmitglied ernannt werden. Darin eingeschlossen sind auch in- und ausländische Gelehrte, die sich auf dem Fachgebiet der Dermatologie und Venerologie besonders ausgezeichnet haben.

${ }^{2}$ Ehrenmitglieder sind von jeglicher Beitragspflicht befreit und im Übrigen den ordentlichen Mitgliedern gleichgestellt.

${ }^{3}$ Die Ernennung erfolgt auf Antrag des Vorstandes durch die Generalversammlung.

\section{Art. 5 Ordentliche Mitglieder}

${ }^{1}$ Als ordentliche Mitglieder werden Ärzte aufgenommen, die den eidgenössischen Facharzttitel Dermatologie und Venerologie erworben haben oder einen ausländischen gleichwertigen, vom Bundesamt für Gesundheit anerkannten Facharzttitel für Dermatologie und Venerologie besitzen.

${ }^{2}$ Wer als ordentliches Mitglied der SGDV beitreten will, hat ein schriftliches Aufnahmegesuch an den Präsidenten zuhanden des Vorstandes zu richten. Dem Gesuch sind ein Lebenslauf, Foto und die Empfehlung von zwei ordentlichen Mitgliedern beizulegen. Der Vorstand prüft die Gesuche und entscheidet über die Aufnahme oder Nichtaufnahme eines Neumitglieds. Der Entscheid über die Ablehnung eines Aufnahmegesuches kann nicht weiter gezogen werden.

${ }^{3}$ Die ordentlichen Mitglieder können nach Aufgabe ihrer beruflichen Tätigkeit Passivmitglieder werden.

\section{Art. 6 Ausserordentliche Mitglieder}

${ }^{1}$ Als ausserordentliche Mitglieder können aufgenommen werden:

- eidgenössisch diplomierte Ärztinnen und Ärzte sowie solche mit einem äquivalenten, anerkannten Diplom und Naturwissenschafter mit Hochschulabschluss, die in Kontakt mit der Dermatologie und Venerologie stehen;

- Oberärzte und Assistenten der schweizerischen dermatologischen Universitätskliniken, Polikliniken und Spezialabteilungen der Kantonsspitäler;

- anerkannte Gelehrte des In- und Auslandes, die besonderes Interesse an den wissenschaftlichen Tätigkeiten der Gesellschaft bekunden.

${ }^{2}$ Für die Aufnahme eines ausserordentlichen Mitgliedes gelten die gleichen Bedingungen wie für ordentliche Mitglieder. Art. 5 Abs. 2 ist analog anwendbar.

${ }^{3}$ Die Mitgliedschaft der Oberärzte und Assistenten ohne eidgenössischen Titel für Dermatologie und Venerologie erlischt mit der Beendigung ihres Arbeitsverhältnisses mit den genannten Instituten.

${ }^{4}$ Ausserordentliche Mitglieder haben in der Generalversammlung kein Stimm- und Wahlrecht, aber eine beratende Stimme.

${ }^{5}$ Mit dem Erwerb des eidgenössischen Titels für Dermatologie und Venerologie wird ein ausserordentliches Mitglied automatisch ordentliches Mitglied gemäss Art. 5.

${ }^{6}$ Ausserordentliche Mitglieder werden nach Aufgabe ihrer Praxistätigkeit zu Passivmitgliedern.

${ }^{7} \mathrm{Zu}$ ausserordentlichen Mitgliedern können ebenfalls juristische Personen, Institute, dermatologische Kliniken usw. ernannt werden, welche die Aufnahmebedingungen der anderen Mitgliederkategorien nicht erfüllen. 


\section{Art. 7 Korrespondierende Mitglieder}

$\mathrm{Zu}$ korrespondierenden Mitgliedern können anerkannte Gelehrte des In- und Auslandes ernannt werden, die besonderes Interesse an den wissenschaftlichen Tätigkeiten der SGDV zeigen. Korrespondierende Mitglieder entrichten keinen Mitgliederbeitrag und erhalten die Einladungen $\mathrm{zu}$ den wissenschaftlichen Veranstaltungen.

\section{Art. 8 Passivmitglieder}

Passivmitglieder sind ordentliche und ausserordentliche Mitglieder, die ihre berufliche Tätigkeit aufgegeben haben.

\section{Art. 9 Beendigung der Mitgliedschaft}

${ }^{1}$ Die Mitgliedschaft wird durch Tod, Austritt oder Ausschluss beendet.

${ }^{2}$ Der Austritt kann schriftlich unter Einhaltung einer Frist von 6 Monaten auf das Ende des Kalenderjahres an den Vorstand erklärt werden.

${ }^{3}$ Die Generalversammlung kann den Ausschluss eines Mitgliedes beschliessen, wenn

- das Mitglied seine statutarischen, insbesondere finanziellen Verpflichtungen gegenüber der SGDV nicht erfüllt, insbesondere bei Nichtbezahlen des Jahresbeitrages trotz zweimaliger schriftlicher Aufforderung durch den Quästor;

- das Mitglied dem Zweck und den Grundsätzen der SGDV und der FMH zuwiderhandelt;

- wenn von Seiten einer kantonalen Ärztegesellschaft gegen ein Mitglied disziplinarische Massnahmen getroffen werden.

${ }^{4}$ Der Ausschluss erfolgt auf Antrag des Ehrenrates. Der Antrag muss unter Nennung des Namens des betroffenen Mitgliedes auf der Traktandenliste der Generalversammlung aufgeführt werden.

${ }^{5}$ Der Ausschlussantrag muss in der Generalversammlung von einem Mitglied des Ehrenrates begründet werden, und dem betroffenen Mitglied ist Gelegenheit zu einer Stellungnahme zu geben. Anschliessend beschliesst die Generalversammlung über den Ausschlussantrag. Für einen Ausschluss ist eine Zustimmung der Mehrheit von zwei Dritteln der Stimmen der anwesenden stimmberechtigten Mitglieder erforderlich. Der Beschluss über den Ausschluss wird dem betroffenen Mitglied schriftlich ohne Angabe einer Begründung mitgeteilt.

${ }^{6}$ In allen Fällen, in denen der Ausschluss eines Mitgliedes in Betracht gezogen wird, muss das Mitglied zuvor vom Ehrenrat angehört werden.

${ }^{7}$ Austritt und Ausschluss schliessen den Verzicht auf die der SGDV geleisteten Beiträge und auf alle Ansprüche auf das Gesellschaftsvermögen in sich.

\section{Art. 10 Rechte}

${ }^{1}$ Die ordentlichen Mitglieder und Ehrenmitglieder und die Mitglieder der ständigen Kommissionen haben - unabhängig von ihrem Status - folgende Rechte:

a) Stimm- und Wahlrecht;

b) Benützung der Dienstleistungen der SGDV, insbesondere den Besuch der im Rahmen der SGDV organisierten Fortbildungskurse $\mathrm{zu}$ vorteilhaften Bedingungen (Jahresversammlung, Frühlingskolloquium und andere Kurse);

${ }^{2}$ Ausserordentliche Mitglieder haben kein Stimm- und Wahlrecht, können aber die unter Art. 10 Abs. 1b erwähnten Dienstleistungen benützen.

${ }^{3}$ Passiv- und korrespondierende Mitglieder haben kein Stimmund Wahlrecht. An den unter Art. 10 Abs. 1b erwähnten Dienstleistungen können sie nur unter gewissen Bedingungen teilnehmen. Der Vorstand erlässt ein Reglement.

\section{Art. 11 Pflichten}

Die Mitglieder sind verpflichtet, die Statuten der SGDV, die Standesordnung der FMH sowie alle weiteren verbindlichen Beschlüsse zu befolgen. Sie bezahlen die für sie zutreffenden Mitgliederbeiträge (Art. 12).

\section{Finanzielle Bestimmung und Haftung}

\section{Art. 12 Finanzquellen}

Die Finanzquellen der SGDV sind:

${ }^{1}$ a) Der ordentliche Jahresbeitrag für die SGDV wird durch die Generalversammlung auf Vorschlag des Vorstandes jährlich festgelegt. Er darf den maximalen Betrag von CHF. 1200.- pro Mitglied nicht überschreiten. Bei der Festlegung des Betrages wird Rücksicht genommen auf die Art der Mitgliedschaft, die Tätigkeit des Mitglieds und auf seine Berufsausübungsbewilligung.

b) Erträge, namentlich aus Veranstaltungen und Publikationen der SGDV;

c) Schenkungen und Vermächtnisse.

2 Der Vorstand kann aus wichtigen Gründen den Beitrag auf Gesuch hin ganz oder teilweise erlassen. Das Gesuch muss begründet werden.

${ }^{3}$ Der Vorstand erlässt ein Reglement über die Beiträge.

\section{Art. 13 Haftung}

Für die Verbindlichkeiten der SGDV haftet allein das Vereinsvermögen. Die Mitglieder sind nur für einen Beitrag in der Höhe ihres Jahresbeitrages persönlich haftbar.

\section{Organe der SGDV}

\section{Allgemeines}

Art. 14 Organe und Amtsdauer

${ }^{1}$ Die Organe der SGDV sind:

- die Generalversammlung;

- der Vorstand;

- der Ausschuss des Vorstandes;

- der Ehrenrat;

- die Rechnungsrevisoren;

- der erweiterte Vorstand;

- das Generalsekretariat.

${ }^{2}$ Die Mitglieder des Vorstandes (mit Ausnahme der Vertreter der fünf dermatologischen Universitätskliniken), des Ehrenrates sowie die beiden Rechnungsrevisoren werden an der Generalversammlung für eine Amtsperiode von zwei Jahren gewählt. Eine Wiederwahl für zwei weitere Amtsperioden ist zulässig. Der Präsident kann nur für eine einzige weitere Amtsperiode wieder gewählt werden.

${ }^{3}$ Die Amtsdauer der fünf Klinikdirektoren der dermatologischen Universitätskliniken ist unbeschränkt und endet mit der Aufgabe ihrer Tätigkeit als Klinikvorsteher.

${ }^{4}$ Für während der Amtsperiode ausscheidende Mitglieder des Vorstandes und des Ehrenrates sowie Rechnungsrevisoren sind an der nächstfolgenden Generalversammlung für den Rest der Amtsperiode Ersatzmitglieder zu wählen. 


\section{Die Jahres- und Generalversammlung}

\section{Art. 15 Allgemeines}

${ }^{1}$ Die Jahresversammlung besteht aus der Generalversammlung und einem wissenschaftlichen Teil.

${ }^{2}$ Die ordentliche Generalversammlung findet während der Jahresversammlung statt. Der Termin wird spätestens zwölf Wochen vorher bekannt gegeben. Der Vorstand lädt die Mitglieder mindestens vier Wochen vorher schriftlich unter Beilage der Traktandenliste ein.

\section{Art. 16 Durchführung der Generalversammlung}

${ }^{1}$ Der Präsident oder der Vizepräsident führt den Vorsitz.

2 Jede Generalversammlung ist beschlussfähig. Stimmberechtigt sind alle anwesenden stimmberechtigten Mitglieder. Jedes Mitglied hat nur eine Stimme, die mit einer vor der Versammlung abgegebenen Stimmkarte ausgeübt wird.

${ }^{3}$ Die Versammlung kann nur über Traktanden Beschluss fassen, die mit der Einladung bekannt gegeben worden sind, ausser über den Antrag auf Durchführung einer ausserordentlichen Generalversammlung.

${ }^{4}$ Die Generalversammlung wählt in offener Abstimmung die erforderliche Anzahl Stimmenzähler.

${ }^{5}$ Bei Abstimmungen entscheidet das einfache Mehr der Stimmenden (der anwesenden stimmberechtigten Mitglieder); der Vorsitzende stimmt nicht mit, bei Stimmengleichheit hat er den Stichentscheid. Stimmenthaltungen, bei geheimen Abstimmungen leere oder ungültige Stimmzettel, werden für die Ermittlung des einfachen Mehrs nicht mitgezählt. Das qualifizierte Mehr nach Massgabe dieser Statuten bleibt vorbehalten.

${ }^{6}$ Bei Wahlen entscheidet das einfache Mehr der Stimmenden (der anwesenden stimmberechtigten Mitglieder). Vom zweiten Wahlgang an kommen keine neuen Kandidaten mehr in die Wahl. Bei jedem neuen Wahlgang scheidet der Kandidat mit der vorher geringsten Stimmenzahl aus; bei Stimmengleichheit wird der ausscheidende Kandidat durch eine Stichwahl bestimmt. Führt die Stichwahl wieder zu Stimmengleichheit, entscheidet das Los. Stimmenthaltungen, bei geheimen Abstimmungen leere oder ungültige Stimmzettel, zählen nicht für die Berechnung des Mehrs.

${ }^{7}$ Vorbehältlich abweichender gesetzlicher oder statutarischer Bestimmungen trifft die Gesellschaft ihre Wahlen und Beschlüsse in der Regel in offener Abstimmung. Der Vorsitzende hat den Stichentscheid.

\section{Art. 17 Anträge zuhanden der Generalversammlung}

1 Jedes Mitglied kann spätestens bis acht Wochen vor der Generalversammlung dem Präsidenten schriftlich Anträge zuhanden der Generalversammlung einreichen.

\section{Art. 18 Befugnisse und Traktanden}

Die Generalversammlung hat folgende Befugnisse:

a) Genehmigung der Jahresberichte des Präsidenten der Gesellschaft;

b) Genehmigung der Jahresrechnung und des Budgets;

c) Wahl des Präsidenten, des Vorstandes und der Rechnungsrevisoren;

d) Entlastung des Präsidenten, des Vorstandes, der Rechnungsrevisoren;

e) Wahl des Ehrenrates und der ständigen Kommissionen;

f) Entscheid über den Ausschluss eines Mitgliedes;

g) Entscheid über alle Gegenstände, die ihr durch Gesetz und Statuten vorbehalten sind, durch den Vorstand vorgelegt werden und über alle fristgerecht eingereichten Anträge.

\section{Art. 19 Ausserordentliche Generalversammlungen}

1 Ausserordentliche Generalversammlungen finden auf Beschluss des Vorstandes oder auf Verlangen von einem Fünftel der stimmberechtigten Mitglieder statt. Die Einladung mit Angabe der Traktanden muss mindestens zehn Tage vor dem Termin unter Angabe des Verhandlungsgegenstandes den Mitgliedern schriftlich zugestellt werden.

${ }^{2}$ Der Vorstand kann mit der Mehrheit von drei Viertel seiner Mitglieder beschliessen, dass die Beschlussfassung einer ausserordentlichen Generalversammlung auf dem Zirkularweg erfolgt.

\section{Der Vorstand}

\section{Art. 20 Zusammensetzung und Wahl}

${ }^{1}$ Der Vorstand besteht mindestens aus 15 Mitgliedern und setzt sich namentlich wie folgt zusammen:

- den Klinikdirektoren der fünf dermatologischen Universitätskliniken;

- aus acht frei praktizierenden Dermatologen;

- zwei Vertretern der nicht universitären Kliniken;

- aus dem Präsident-elect, insofern einer ernannt wurde.

${ }^{2}$ Der Vorstand wird, mit Ausnahme der Klinikdirektoren der Universitätskliniken, von der Generalversammlung gewählt.

${ }^{3}$ Bei der Wahl der Vorstandsmitglieder ist nach Möglichkeit auf eine angemessene Vertretung der Sprachregionen des Landes zu achten. Die freigewordenen Vorstandsmandate werden ein Jahr vorher an der Generalversammlung bekannt gegeben. Die Vorschläge für Kandidaten können bis 6 Monate vor der nächsten Generalversammlung an den Vorstand eingereicht werden.

${ }^{4}$ Auf Vorschlag des Vorstandes wählt die Generalversammlung aus dem Kreis der Vorstandsmitglieder den Präsidenten, der in der Regel dem Vorstand während mindestens einer Amtsperiode als Mitglied angehört haben muss. Das Amt des Präsidenten kann nur während zwei Amtsperioden von je zwei Jahren ausgeübt werden. Vorgesehen ist eine Periode als Präsident-elect während einem Jahr, die ausnahmsweise verlängert werden kann. Die Jahre als Präsident können zusätzlich zu den Jahren im Vorstand absolviert werden.

${ }^{5}$ Die Generalversammlung kann auf Vorschlag des Vorstandes einen Präsident-elect, der als zukünftiger Präsident vorgesehen ist, ein Jahr vor seiner Wahl als Präsidenten wählen. Der Präsidentelect wird, falls er es nicht schon ist, automatisch Vorstandsmitglied.

\section{Art. 21 Zuständigkeit und Kompetenzen}

${ }^{1}$ Der Vorstand leitet die Geschäfte der Gesellschaft und unternimmt alles, was den Zielen der Gesellschaft dient und in ihrem Interesse liegt. Alle Geschäfte, die nicht ausdrücklich nach Gesetz oder Statuten der Generalversammlung vorbehalten sind, werden durch den Vorstand erledigt.

${ }^{2}$ Er kann zur Bearbeitung einzelner Geschäfte Ad-hoc-Kommissionen aus Mitgliedern der Gesellschaft bilden. Der Auftrag und die Zusammensetzung der Ad-hoc-Kommissionen werden vom Vorstand in seinen Protokollen festgelegt.

${ }^{3}$ Der Vorstand legt das Geschäftsjahr fest. Bis zu einer Gesamtsumme von CHF 10 000.- pro Jahr kann er dringliche Ausgaben, die im Voranschlag nicht aufgeführt sind, beschliessen.

${ }^{4}$ Der Vorstand bestimmt unter seinen Mitgliedern den Vizepräsidenten und den Kassier.

${ }^{5}$ Der Vorstand wählt den Generalsekretär.

${ }^{6}$ Der Vorstand legt die Prüfungsgebühr zum Fachexamen fest. Diese Gebühren sollen Kosten deckend sein. 
Art. 22 Organisation und Aufgaben der einzelnen Vorstandsmitglieder

${ }^{1}$ Der Präsident beruft den Vorstand ein, wenn es die Geschäfte erfordern. Drei Vorstandsmitglieder können die Einberufung ebenfalls verlangen.

2 Der Präsident, im Verhinderungsfalle der Vizepräsident, leitet als Vorsitzender die Vorstandssitzungen. Er vertritt die SGDV nach aussen. Er hat bei Vorstandsentscheiden mit Stimmengleichheit den Stichentscheid.

${ }^{3}$ Der Quästor ist verantwortlich für das Rechnungswesen. Er legt der Generalversammlung Jahresrechnung und Budget vor.

\section{Art. 23 Zeichnungsberechtigung}

Die rechtsverbindliche Unterschrift führen der Präsident oder der Vizepräsident kollektiv zu zweien mit einem Mitglied des Ausschusses oder dem Quästor.

\section{Art. 24 Ausschuss}

${ }^{1}$ Der Vorstand ernennt einen Ausschuss, bestehend mindestens aus dem Präsidenten, dem Vizepräsidenten und einem Vertreter einer dermatologischen Universitätsklinik. Er übernimmt die exekutive Leitung der SGDV, entscheidet und handelt in dringlichen Fällen. Über die getroffenen Massnahmen orientiert er zeitgerecht den Vorstand.

2 Dem Vorstandsausschuss obliegt die Erledigung der laufenden Geschäfte und die Vorbereitung der im Gesamtvorstand zu behandelnden Fragen.

${ }^{3}$ Der Präsident leitet die Sitzung.

\section{Der Ehrenrat}

\section{Art. 25 Zusammensetzung des Ehrenrates}

${ }^{1}$ Der Ehrenrat hat fünf Mitglieder und setzt sich wie folgt zusammen aus:

- einem Präsidenten;

- zwei Vertretern der deutschen und je einem der

französischen und der italienischen Schweiz;

- einem Ersatzmitglied.

2 Der Ehrenrat wird von der Generalversammlung für eine Amtsperiode von zwei Jahren gewählt. Eine Wiederwahl für zwei weitere Amtsperioden ist zulässig.

\section{Art. 26 Zuständigkeit}

Der Ehrenrat hat von sich aus oder auf Antrag des Vorstandes in Fällen einzuschreiten, in denen das Verhalten eines Mitgliedes den guten Ruf und das Ansehen der Gesellschaft zu beeinträchtigen droht.

Der Ehrenrat kann einen Tadel aussprechen oder der Generalversammlung Antrag auf Ausschluss des Mitgliedes stellen. Der Ehrenrat kann die Akten der betroffenen kantonalen Gesellschaft weiterleiten.

\section{Art. 27 Rechtsmittel}

Gegen die Entscheidungen des Ehrenrates sind keine Rechtsmittel möglich.

\section{Die Rechnungsrevisoren}

Die Generalversammlung wählt zwei Rechnungsrevisoren, welche die Rechnungslegung des Quästors überprüfen und der Generalversammlung Bericht und Antrag über die Genehmigung oder Ablehnung der Jahresrechnung stellen.

\section{Der erweiterte Vorstand}

\section{Art. 28 Organisation}

Der Vorstand, der Präsident des Ehrenrates, die Präsidenten der ständigen Kommissionen, die Präsidenten der Arbeitsgruppen und die Präsidenten der regionalen Gesellschaften treffen sich einmal jährlich zu einem Informationsaustausch, grundsätzlich anlässlich der Jahresversammlung.

\section{Das Generalsekretariat}

\section{Art. 29 Zusammensetzung und Zuständigkeit}

${ }^{1}$ Der Vorstand kann gewisse Aufgaben einem Generalsekretariat delegieren, welches die Tätigkeiten des Präsidenten, des Ausschusses und des Vorstandes unterstützt. Es besteht aus dem Generalsekretär und einem Verwaltungssekretariat.

2 Der Generalsekretär arbeitet auf Mandatsbasis.

3 Die Höhe seiner Entschädigung wird vom Vorstand beschlossen.

\section{Die ständigen Kommissionen}

\section{Art. 30 Allgemeines}

${ }^{1}$ Die folgenden Kommissionen bestehen ständig, erstatten dem Vorstand mindestens einmal jährlich Bericht und bestehen aus mindestens 5 Mitgliedern. Ein Vorstandsmitglied sollte jeweils in jeder Kommission vertreten sein. Die Mitglieder werden von der Generalversammlung für eine Amtsperiode von zwei Jahren gewählt. Eine Wiederwahl für zwei weitere Amtsperioden ist zulässig.

2 Jede Kommission wählt einen Präsidenten für eine Amtsperiode. Eine Wiederwahl für eine weitere Amtsperiode ist zulässig. Die Jahre als Präsident können zusätzlich zu den Jahren in der Kommission absolviert werden.

${ }^{3}$ Der Vorstand legt in Rücksprache mit den Kommissionsmitgliedern die Reglemente über die Tätigkeit der Kommissionen fest.

${ }^{4}$ In ständigen Kommissionen können ordentliche, ausserordentliche und Passivmitglieder Einsitz nehmen.

\section{Art. 31 Kommission zur Wahrung der Standesinteressen}

${ }^{1}$ Der Präsident der Kommission muss gleichzeitig Vorstandsmitglied sein.

${ }^{2}$ Die Kommission hat die Aufgabe, Tariffragen zu bearbeiten und die standespolitischen Interessen der Praktiker zu vertreten.

\section{Art. 32 Fachexamenskommission}

${ }^{1}$ Die Fachexamenskommission hat den Auftrag, das jährliche Fachexamen in Dermatologie und Venerologie durchzuführen gemäss der Fort- und Weiterbildungsordnung der FMH und dem Weiterbildungsprogramm der SGDV. Ihr Präsident muss Chef einer dermatologischen Universitätsklinik oder ein Arzt mit Rang eines Professoren einer dermatologischen Universitätsklinik sein.

${ }^{2}$ Die Prüfungsgebühren werden vom Vorstand festgelegt. Die Prüfungsgebühren müssen die Kosten decken. 
Art. 33 Kommission für Qualitätssicherung

${ }^{1}$ Die Kommission hat den Auftrag, Programme für die Qualitätssicherung gemäss den geltenden Bestimmungen der FMH vorzuschlagen und Experten mit deren Durchführung zu beauftragen.

${ }^{2}$ Die Kosten der Qualitätssicherungsprogramme müssen vom Vorstand genehmigt werden. Ihre Finanzierung wird von Fall zu Fall beschlossen.

\section{Art. 34 Kommission für Weiter- und Fortbildung}

${ }^{1}$ Die Kommission für Weiter- und Fortbildung (KWF) hat den Auftrag, die Ausarbeitung und permanente Revision des Weiterund Fortbildungsprogrammes der SGDV gemäss der geltenden Weiter- und Fortbildungsordnung der FMH durchzuführen. Sie kann Arbeitsgruppen einsetzen.

${ }^{2}$ Der KWF obliegt es, wissenschaftliche Veranstaltungen, die nicht im Weiter- und Fortbildungsprogramm erwähnt sind, hinsichtlich der Fortbildung zu evaluieren und den Mitgliedern der SGDV Atteste über geleistete Fortbildung auszustellen. Jedes Mitglied der SGDV kann innerhalb von 30 Tagen Einspruch gegen eine Entscheidung der KWF beim Ehrenrat erheben.

${ }^{3}$ Die fünf Mitglieder werden auf Antrag des Vorstandes von der Generalversammlung gewählt. Ein Mitglied muss den Rang eines Professors haben. Es ist nicht zulässig, gleichzeitig Mitglied des Ehrenrates und der KWF zu sein.

\section{Art. 35 Medienkommission}

Die Medienkommission überwacht die Aktivitäten der Mitglieder in den Medien bzw. die Darstellung der SGDV in der Öffentlichkeit. Sie ist für die Führung der Homepage zuständig.

\section{Art. 36 Therapeutikakommission}

${ }^{1}$ Die Therapeutikakommission hat den Auftrag, die Wirksamkeit, die Zweckmässigkeit und die Wirtschaftlichkeit von eingesetzten Dermatalogika zu überwachen.

${ }^{2}$ Im Namen der SGDV koordiniert sie die Kommunikation zwischen dem BAG, dem BSV, Swissmedic und den Pharma-Firmen und ist deren direkter Ansprechpartner.

${ }^{3}$ Mindestens 2 Vertreter der Kliniken sind in der Therapeutikakommission vertreten.

\section{Arbeitsgruppen}

\section{Art. 37 Funktion}

Arbeitsgruppen haben zum Ziel, besonderen Themen der Dermatologie und Venerologie nachzugehen und sie zu fördern.

\section{Art. 38 Zusammensetzung}

Arbeitsgruppen können von ordentlichen und ausserordentlichen Mitgliedern mit der Zustimmung des Vorstandes frei gebildet werden.

\section{Entschädigung der Mandatsträger}

\section{Art. 39 Entschädigung des Vorstandes und der Kommissions-} mitglieder

${ }^{1}$ Der Präsident der SGDV erhält eine jährliche Pauschalentschädigung, die durch die Generalversammlung bestätigt wird.

${ }^{2}$ Die Mitglieder des Vorstandes und der Kommissionen erhalten ein Sitzungsgeld.

${ }^{3}$ Die Sekretariatskosten und Reisespesen der Mitglieder des
Vorstandes und der Kommissionen werden von der SGDV übernommen.

${ }^{4}$ Der Vorstand erarbeitet ein Reglement über die Entschädigungen, welches von der Generalversammlung zu genehmigen ist.

\section{Statutenänderung}

\section{Art. 40 Anträge}

Statutenänderungen können nur in einer ordnungsgemäss einberufenen Generalversammlung beschlossen werden. Der Änderungsantrag ist der Einladung zur Versammlung beizulegen. Anträge auf Änderung der Statuten sind dem Vorstand mindestens zwei Monate vor der Versammlung schriftlich einzureichen. Eine Statutenänderung erfordert die Zustimmung von zwei Dritteln der Stimmen der anwesenden stimmberechtigten Mitglieder.

\section{Auflösung der Gesellschaft}

Art. 41 Verfahren, Vermögen

${ }^{1}$ Die Auflösung der SGDV ist durch die Generalversammlung $\mathrm{zu}$ beschliessen. Erforderlich ist die Zustimmung von drei Vierteln der abgegebenen Stimmen der anwesenden stimmberechtigten Mitglieder.

${ }^{2}$ Das zu diesem Zeitpunkt vorhandene Vereinsvermögen wird nach Erfüllung aller Verpflichtungen einer schweizerischen Bank zur treuhänderischen Verwaltung übergeben. Im Falle, dass später eine neue Gesellschaft für Dermatologie und Venerologie mit vergleichbarer Zweckbestimmung gegründet wird, kann diese der Treuhänderin die Aushändigung des Vermögens samt aufgelaufener Zinsen beantragen.

\section{Schlussbestimmungen}

Diese Statuten wurden an der Generalversammlung vom 9. September 2005 beschlossen und sofort in Kraft gesetzt. Sie ersetzen alle früheren Statuten der SGDV. Mit Inkraftsetzung der revidierten Statuten sind alle damit in Widerspruch stehenden früheren Gesellschaftsbeschlüsse aufgehoben.

Massgebend ist der deutsche Text der vorliegenden Statuten.
Der Präsident:
Der Vize-Präsident:
Dr. Félix Gueissaz Prof. Dr. Alfred Eichmann

La version française sera publieé dans un prochain numéro de Dermatologica Helvetica. 


\section{Inhaltsverzeichnis}

\section{Name, Sitz und Zweck}

Art. 1 Name und Sitz

Art. 2 Zweck

\section{Mitgliedschaft}

Art. 3 Mitgliederkategorien

Art. 4 Ehrenmitglieder

Art. 5 Ordentliche Mitglieder

Art. 6 Ausserordentliche Mitglieder

Art. 7 Korrespondierende Mitglieder

Art. 8 Passivmitglieder

Art. 9 Beendigung der Mitgliedschaft

Art. 10 Rechte

Art. 11 Pflichten

\section{Finanzielle Bestimmung und Haftung}

Art. 12 Finanzquellen

Art. 13 Haftung

\section{Organe der SGDV}

1. Allgemeines

Art. 14 Organe und Amtsdauer

2. Die Jahres- und Generalversammlung

Art. 15 Allgemeines

Art. 16 Durchführung der Generalversammlung

Art. 17 Anträge zuhanden der Generalversammlung

Art. 18 Befugnisse und Traktanden

Art. 19 Ausserordentliche Generalversammlungen

3. Der Vorstand

Art. 20 Zusammensetzung und Wahl

Art. 21 Zuständigkeit und Kompetenzen

Art. 22 Organisation und Aufgaben der einzelnen

Vorstandsmitglieder
Art. 23 Zeichnungsberechtigung $\quad 26$

Art. 24 Ausschuss 26

4. Der Ehrenrat

Art. 25 Zusammensetzung des Ehrenrates 26

Art. 26 Zuständigkeit 26

Art. 27 Rechtsmittel 26

5. Die Rechnungsrevisoren 26

6. Der erweiterte Vorstand 26

Art. 28 Organisation 26

7. Das Generalsekretariat

Art. 29 Zusammensetzung und Zuständigkeit 26

V. Die ständigen Kommissionen 26

Art. 30 Allgemeines 26

Art. 31 Kommission zur Wahrung der Standesinteressen 26

Art. 32 Fachexamenskommission 26

Art. 33 Kommission für Qualitätssicherung 29

Art. 34 Kommission für Weiter- und Fortbildung 29

Art. 35 Medienkommission 29

Art. 36 Therapeutikakommission 29

VI. Arbeitsgruppen 29

Art. 37 Funktion 29

Art. 38 Zusammensetzung 29

VII. Entschädigung der Mandatsträger 29

Art. 39 Entschädigung des Vorstandes und der

VIII. Statutenänderung 29

Art. 40 Anträge 29

IX. Auflösung der Gesellschaft 29

Art. 41 Verfahren, Vermögen 29

X. Schlussbestimmungen 29

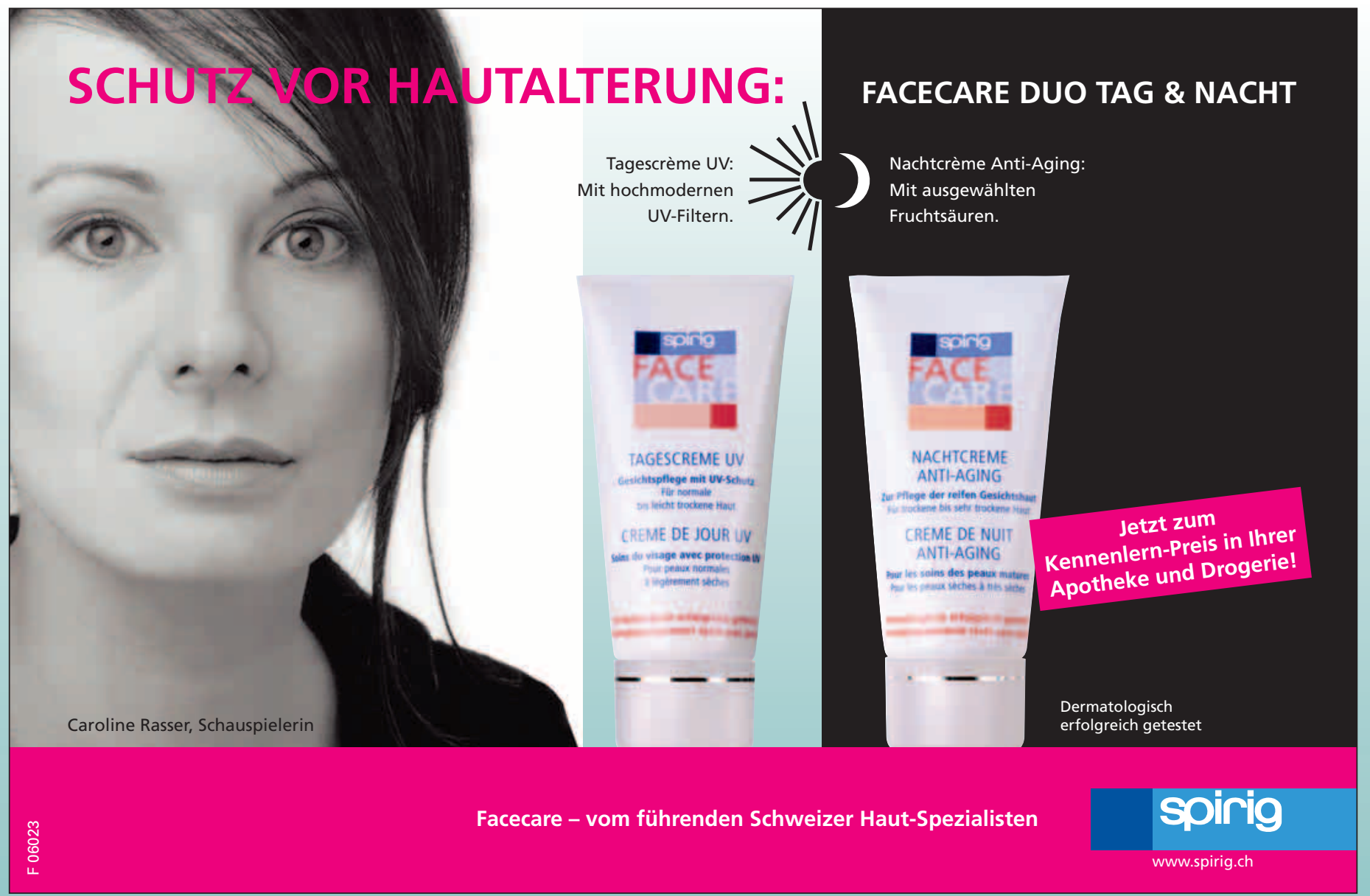

